\title{
Qualità e sostenibilità in sanità
}

\author{
Giuseppe Quintaliani ${ }^{1}$, Maria Luisa Standoli ${ }^{2}$, Carlo Giammarioli ${ }^{3}$ \\ ${ }^{1}$ Coordinatore Governo Clinico SIN \\ ${ }^{2}$ Scuola di specializzazione in Nefrologia, Perugia \\ ${ }^{3} \mathrm{SC}$ Nefrologia e Dialisi, Ospedale Santa Maria della Misericordia, Perugia
}

QUALITY AND SUSTAINABILITY OF THE HEALTH CARE SYSTEM

Abstract. There are different opinions about the quality of our national health system (NHS): in some rankings it is the second in Europe, while in others it is the $21 \mathrm{st}$. The use of cost-related indicators, instead of clinical-related ones, leads to a severe downgrade of the Italian NHS in the European context.

According to the demographic changes of the population, consisting more and more of old people and chronically-ill patients, it is necessary to develop and use clinical process indicators proposed by the scientific societies, thus abandoning the Hospital Discharge File as the clinical quality assessment system: the reduction of the costs shall not be considered a good result without an increase in clinical effectiveness.

The use of information flows through the informatics improvement of the so-called "big data", and the crosscheck of data may be a great help for the development of this process. The Italian NHS will be saved by a strong reduction of costs that starts from the diagnostic appropriateness and clinical efficacy.

Key words: Management, Health policy, Health economics

Conflict of interest: None.

Financial support: None.

Accettato: 1 Febbraio 2014

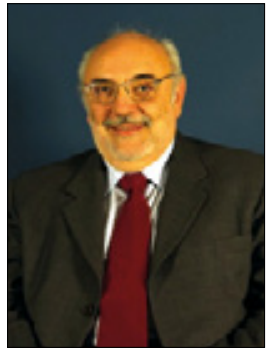

$\mathrm{Ci}$ sono diverse interpretazioni sulla qualità del nostro SSN che, proprio in questi giorni, compie il $35^{\circ}$ compleanno. Il famoso report dell'OCSE, così tanto citato dai nostri politici e amministratori, che poneva la nostra sanità al secondo posto in Europa (1), era dovuto al fatto che, pur essendo un SS parco in spese, i risultati erano abbastanza buoni in rapporto al costo, mentre nulla diceva Giuseppe Quintaliani rispetto alla qualità clinica ottenuta. $\mathrm{Da}$ allora, ci sono stati altri report (2) e altre statistiche non incoraggianti e brillanti per la posizione che l'Italia sembra occupare nelle classifiche internazionali.

Ultimo arrivato, un report svedese del 2013 pone la sanità italiana in una posizione molto critica e molto lontana dai vertici (3). Afferma, infatti, il Sole 24 ore Sanità: "L'amara verità per l'Italia è venuta a galla senza pietà: siamo decimi per la qualità della nostra sanità pubblica, undicesimi per $i$ «particolari vantaggi» del SSN e, addirittura, tredicesimi per l'equità nell'offerta dei servizi".

In mezzo ci sono altri report internazionali che affossano la nostra sanità, come l'Euro Health Consumer Index (EHCI)
(4), con la valutazione di 34 sistemi sanitari europei, giunto nel 2013 alla nona edizione, che vede in testa alla classifica, con 872 punti, l'Olanda, mentre l'Italia, con 623 punti, si trova al $21^{\circ}$ posto.

Non tutte le statistiche ci vedono in una così brutta posizione; in effetti, altri report, come il Global Burden of Disease Study (5) del 2010 sul Lancet, promuovono, invece, la sanità italiana (6). Del tutto recentemente, una statistica italiana pone cinque Regioni al vertice della sanità italiana (7). Il report si occupa specificatamente di problemi economici, ma pone attenzione anche alla fornitura di prestazioni all'interno dei LEA. Anche in questo caso, gli indicatori e i criteri usati hanno suscitato dubbi e incertezze, con un acceso dibattito tra gli esperti, in quanto tutti questi parametri sono presi dalle SDO (Schede di Dimissione Ospedaliera) dell'anno 2011, Progetto Agenas (8). Prendiamo, per esempio, uno degli indicatori: "percentuali interventi per frattura del femore operati entro due giorni". È vero che tale obiettivo, se raggiunto, porta risparmi economici ed è frutto di un'attenta organizzazione e di precisi percorsi, ma è anche vero che nulla ci dice sulle infezioni, sui sanguinamenti e sul consumo di sangue, sulle eventuali embolie e sulle complicanze in genere dell'intervento e ancora NULLA ci dice della qualità professionale dell' equipe chirurgica (ortopedica, in questo caso). Ė del tutto evidente 
la differenza di valutazione tra chi vuole essere efficiente e chi vuole prima e soprattutto efficacia e correttezza.

Lo stesso lo si può dire per i trattamenti medici dove l'area di incertezza è ancora più ampia. Per esempio, un paziente ricoverato in cardiologia che rientra dopo un mese in medicina per un problema legato al diabete è stato curato bene? Il numero di giorni di degenza in cardiologia entro la media è un buon risultato se non c'è stato il tempo di valutare anche i problemi connessi allo stato diabetico? E la funzione renale è stata sufficientemente indagata? Quanti ricoveri ripetuti si sono verificati?

Se ne deduce, quindi, che il sistema sia molto difficile da valutare e da misurare a meno che:

non si abbandoni il solo costo come strumento di valutazione;

non si ricorra a indicatori di processo clinici dettati dalle varie società scientifiche.

L'abbattimento del costo non può essere un risultato, ma solo un vincolo; in altre parole, si deve PRIMA ottenere un risultato vero in termini di salute e solo POI lo si deve ottenere al minor costo possibile (in farmaco economia si parla di valutazione costo eficacia).

Per capire, quindi, se un costo è stato giusto o meno, si deve valutare la prestazione. Gli indicatori di esito sono i migliori, ma è necessario molto tempo per ottenerli, e, se vogliamo che siano corretti (tipicamente la mortalità), devono essere esenti da errori metodologici. Per esempio, Agenas considera la sopravvivenza all'infarto, ma non misura la gravità dell'episodio né i fattori complicanti (diabete, insufficienza renale, età, aterosclerosi, ecc.). Quindi, si corre il rischio di premiare cardiologie che vedono infarti poco gravi penalizzando reparti di alta complessità dotati anche di emodinamica che sono gravati di mortalità non per incapacità, ma solo per la gravità della casistica.

È necessario, quindi, avere a disposizione degli indicatori che veramente fotografino la realtà clinica, e l'interlocutore non può essere il direttore generale a cui spetta il compito di osservarli, ma deve essere la società scientifica che deve stenderli. Come succede nei protocolli di ricerca, il controllato deve essere diverso dal controllore! Inoltre, attualmente, l'uso oculato dei dati computerizzati (i cosiddetti big data) potrebbe permettere la valutazione dell'efficacia clinica della terapia. Viviamo nell'epoca delle malattie croniche ed è fondamentale sapere se, all'uscita da un reparto clinico, si sia effettuata una valutazione del diabete, della funzione renale e della situazione cardiologica tale da ridurre $\mathrm{i}$ rischi connessi a tali patologie. In caso contrario, avremo risolto magari il problema intercorrente, ma avremo gettato i semi per futuri ricoveri e complicazioni che non faranno altro che aumentare i costi. I costi non sono quelli dell'anno in corso ma sono frutto di una strategia complessa e pluriannuale. Se è vero che dobbiamo rendere 2.000 miliardi di euro di debito a cui vanno aggiunti almeno 80 miliardi annuali di interessi, è ovvio che l'orizzonte temporale si sposta a circa 10 anni e non all'anno in corso o al prossimo e che, se vorremo avere successo, dovremo mettere in atto strategie che riducano nel medio-lungo raggio le spese, riducendo le complicazioni e il bisogno di cure. Oppure ci dicano che alcune fette della popolazione affette da alcuni tipi di patologie non saranno più trattate entro 3-4 anni (questa è l'opinione di Ricciardi, Prof. di management sanitario alla Cattolica, che pone la fine del SSN nel 2017). Se la popolazione più povera si ammala di più e tende a non curarsi (come dice l'ISTAT nell'ultimo rapporto del Dicembre del 2013), allora dovremo studiare nuove forme di assistenza per scongiurare futuri peggioramenti che, attualmente, sono a carico della popolazione. Basti ricordare che le visite specialistiche sono pagate di tasca propria nel $54 \%$ dei casi e che sono circa 1 milione e mezzo le badanti che assistono i nostri vecchi (e i cui proventi, circa 5 miliardi all'anno, se ne vanno all'estero). Si stima che i pazienti con una malattia cronica siano pari al $30 \%$ della popolazione, equivalenti a circa 18 milioni (in Umbria sono circa 270.000 con circa 45.000 nefropatici); lo stesso rapporto ci dice che per un MMG massimalista ci sono 450 cronici. Inoltre, circa 2.5 milioni (4\% della popolazione) sono anziani non autosufficienti (forse ancora di più, secondo la Federanziani, che ne censisce circa 4 milioni) (9); non tutti sono assistiti da badanti e sono a totale carico delle famiglie che, peraltro, per via della crisi, vedono continuamente assottigliarsi i guadagni e, in caso di assistenza all'anziano, anche la possibilità di cercare fonti di reddito alternative.

Questi aspetti economico/sociali potrebbero essere vissuti anche come un'opportunità. Sarebbe lungo affrontare per intero la questione ma il nodo principale è quello di sviluppare degli indicatori di processo validi e validati abbandonando la SDO come sistema di valutazione della qualità clinica. I grandi flussi informatici (i cosiddetti big data) ci possono aiutare. L'incrocio tra i vari database amministrativi, come quelli dei ricoveri, delle esenzioni, della farmaceutica e dei laboratori analisi, è uno strumento potente che può dare grossi e quanto mai validi risultati. La valutazione, per esempio, della glicemia (o, ancora meglio, dell'Hb1Ac) nei pazienti diabetici (basta avere il codice esenzione) ci potrebbe dire come stiamo lavorando sul diabete; la valutazione della funzione renale sempre nel diabetico potrebbe aiutarci a ridurre gli ingressi in dialisi a fronte di una patologia che è al primo posto negli USA e che si avvia a diventare la seconda in Italia e in cui circa la metà dei pazienti non viene indagata per la patologia nefrologica. Ancora più potente sarebbe l'analisi di valutazione della compliance alla terapia e dell'appropriatezza prescrittiva. Come si vede, i risparmi potrebbero essere notevoli e l'impegno in costi potrebbe essere modesto, sempre che ci si rivolga a software house con know-how certificato e a epidemiologi ed esperti di varie patologie con criteri meritocratici.

Misure di accompagnamento con un forte impatto sociale ed economico sono ancora possibili: a) aumentare i letti in RSA, magari private, con stringenti controlli qualitativi; b) aiutare i MMG a effettuare meglio la loro professione chiudendo i piccoli ospedali e riconvertendoli in ambulatori per aggregazioni di MMG con supporti diagnostici (come si possa pretendere che un medico faccia il suo lavoro con il solo fonendoscopio è qualcosa di inimmaginabile); c) 
utilizzare l'informatica come strumento di analisi dei dati clinici e non come strumento gestionale.

Torneremo su questi aspetti ma, se mi si lascia la conclusione da un punto di vista nefrologico, penso che la valutazione della cura del paziente nefrologico debba essere effettuata sulla base di indicatori di qualità stabiliti dalla società scientifica passando attraverso a) la "clinical competence" (fa chi sa fare e lo dimostra), b) l'interazione pubblico-privato DOPO la stesura di adatti indicatori clinici, c) la riduzione dell'ingresso in dialisi potenziando gli ambulatori con la possibilità di vedere il paziente cronico entro tempi ristretti (anche ogni mese, se necessario) e tenendo conto dell'empatia del personale e dell'aderenza alla terapia, d) l'implementazione di adatti percorsi di sostegno alle cure palliative come anche recentemente riconosciuto al congresso della società americana di nefrologia, e) il sostegno della donazione da vivente (proprio in questi giorni uno studio Censis-SIN dimostra l'abbattimento dei costi di circa il 30\%), f) il sostegno del trasferimento dei pazienti nefrologici più complessi in corsie nefrologiche (e quando parlo di pazienti nefrologici non parlo solo di trapianto ma soprattutto di pazienti in fase iniziale di nefropatia in cui un intervento precoce è in grado di ridurre l'ingresso in dialisi (Remuzzi: "remission clinic") e g) la promozione di percorsi clinici concordati e condivisi con i MMG che possano essere artefici del follow-up nefrologico e trovare delle corsie preferenziali di vita e consulenza in caso di nuovi eventi comorbidi e alterazioni non programmate (la maggior parte dei pazienti nefropatici ha una lenta progressione che non prevede interventi particolari, se non un attento follow-up). In conclusione, possiamo dire che il SSN si salverà e ridurrà le spese se le scelte saranno basate sulla clinica e sull'appropriatezza clinica e non gestionale, se le valutazioni saranno effettuate in base a indicatori esterni alle Regioni e ai singoli ospedali e sulla base di indicatori nazionali e clinici, permettendo benchmarking intra ed extraregionali e se i modelli gestionali saranno volti a ridurre i costi futuri derivanti dalle complicanze o da errati o ritardati trattamenti clinici, abbandonando l'inutile e dannosa rincorsa all'abbattimento dei costi fine a se stesso, che non fa altro che spostare nel futuro ben più onerosi e difficili oneri e sacrifici.

\section{Riassunto}

$\mathrm{Ci}$ sono diverse interpretazioni sulla qualità del nostro SSN: per alcuni è al secondo posto in Europa, mentre per altri finiamo al $21^{\circ}$ posto in classifica. Questo per l'utilizzo di diversi indicatori, di cui la maggior parte è basata sul costo e non sulla qualità clinica.

Per la sopravvivenza del nostro SSN, anche in considerazione del cambiamento demografico della popolazione, formata da anziani e malati cronici, è necessario sviluppare e utilizzare indicatori di processo clinici dettati dalle varie società scientifiche, abbandonando la SDO come sistema di valutazione della qualità clinica: l'abbattimento del costo, se non accompagnato all'efficacia clinica, non può essere considerato un risultato.

In questo processo, di grande aiuto possono essere i flussi informatici, i cosiddetti big data, attraverso l'incrocio tra i vari database.

Il SSN si salverà se ridurrà davvero le spese, basando le scelte sulla clinica e sull'appropriatezza diagnostica.

Parole chiave: Farmacoeconomia, Politica sanitaria, $M a-$ nagement

Dichiarazione di conflitto di interessi: Gli Autori dichiarano di non avere conflitto di interesse.

Contributi economici agli Autori: Gli Autori dichiarano di non aver ricevuto sponsorizzazioni economiche per la preparazione dell'articolo.

Indirizzo degli Autori:

Prof. Giuseppe Quintaliani

S.C. Nefrologia e Dialisi

Ospedale S.M. Misericordia

S. Andrea delle Fratte

06156 Perugia

quintaliani@yahoo.it

\section{Bibliografia}

1. The world health report 2000 - Health systems: improving performance, http://www.who.int/whr/2000/en/index.htm.

2. L'Italia virtuosa della spesa sanitaria, http://archivio.lavoce. info/articoli/pagina1002517.html .

3. La ricerca svedese: sanità italiana sotto metà classifica. La Calabria ultima. E il Sud affonda, http://www.sanita.ilsole24ore.c iori om/art/in-europa-e-dal-mondo/2013-07-26/ricerca-svedese-sanita-italiana-105744.php.

4. Euro Health Consumer Index (EHCI), http://www.healthpo- werhouse.com/.

5. Global Burden of Disease Study 2010 Lancet. Published Dec 13, 2012. http://www.thelancet.com/themed/global-burden-of-disease

6. EPICENTRO: Global Burden of Disease Study 2010: promossa la sanità italiana.

7. Costi standard, confermate le cinque Regioni di riferimento, Sole 24 ore Sanità.

8. Programma nazionale, esiti Agenas.

9. http://www.sanitaincifre.it/2014/01/non-autosufficienza-colpisce-4-milioni-di-italiani/. 